\title{
Questes
}

vestes Revue pluridisciplinaire d'études médiévales

$41 \mid 2019$

\section{L'insulte et l'injure}

\section{Préface}

\section{Claude Gauvard}

\section{(2) OpenEdition \\ Journals}

\section{Édition électronique}

URL : http://journals.openedition.org/questes/5460

DOI : 10.4000 /questes.5460

ISSN : 2109-9472

\section{Éditeur}

Les Amis de Questes

\section{Édition imprimée}

Date de publication : 11 novembre 2019

Pagination : 1-9

ISSN : 2102-7188

\section{Référence électronique}

Claude Gauvard, « Préface », Questes [En ligne], 41 | 2019, mis en ligne le 20 décembre 2019, consulté le 25 septembre 2020. URL : http://journals.openedition.org/questes/5460 ; DOI : https://doi.org/ 10.4000 /questes.5460 


\title{
Préface
}

\author{
Claude GAUVARD \\ Université Paris 1 Panthéon-Sorbonne - LaMOP
}

« Ajouter l'insulte à l'injure », cette expression populaire associe les deux mots titres de ce numéro et permet de les englober dans le même schéma de l'outrage, tout en donnant à chacun sa place propre que confirme son étymologie : à l'injure, le franchissement du jus, de la norme par le verbe ou le geste, à l'insulte celui du glissement dans la violence physique du saut, face à face avec l'adversaire ${ }^{1}$. Les deux, quelle que soit la gradation, provoquent des dommages, troublent la paix et imposent une réparation.

Le thème est le fruit d'une historiographie déjà ancienne, une trentaine d'années après la parution en 1993-1994 du numéro de la revue Médiévales, La Renommée ${ }^{2}$ et celui d'Atalaya, L'invective au Moyen Âge. France, Espagne, Italie $^{3}$. Il est très utile de le reprendre à nouveaux frais pour mesurer le chemin parcouru et unir une nouvelle fois les spécialistes de la littérature et de l'histoire du Moyen Âge. Leur collaboration est d'autant plus indispensable que l'injure, sous sa forme verbale et malgré son caractère stéréotypé, véhicule un vocabulaire riche et varié, déclinant des expressions dialectales que les autorités judiciaires ont pu être conduites à traduire. À Amiens, le rédacteur de la charte de commune précise que le « wissot» de l'injure est un « coup», homme marié trompé et cocu; au bailliage de Saint-Pierre-le-Moûtier, que «cornart»

\footnotetext{
${ }^{1}$ Sur la nuance des mots, voir Laurence Rosier, Petit traité de l'insulte, Bruxelles, Labor, coll. « Espace de libertés », 2006, p. 19.

${ }^{2}$ Médiévales, $\mathrm{n}^{\circ} 24$, La renommée, dir. Claude Gauvard, 1993.

${ }^{3}$ L'Invective au Moyen Âge: France, Espagne, Italie. Actes du colloque L'Invective au Moyen Âge, Paris, 4-6 février 1993, dir. Éric Beaumatin et Michel Garcia, Paris, Presses de la Sorbonne Nouvelle, coll. « Atalaya », 1994, qui donne un état de la bibliographie, p. 263-269.
} 
équivaut, selon la coutume du pays, à un « $\operatorname{coup}^{4} »$. Les injures peuvent aussi évoluer dans le temps et par conséquent faire référence au contexte historique : être appelé «Anglais » ou «Bourguignon » prend un sens précis au $\mathrm{XV}^{\mathrm{e}}$ siècle, susceptible de déclencher la vengeance; au même moment, traiter son adversaire de «sorcier» ou de «vieille lisse sorcière» se colore d'une dénonciation qui risque d'être perçue comme irréversible, car la justice s'est emparée de la sorcellerie populaire et peut conduire au bûcher.

Jusqu'aux années 1990, l'injure était plutôt perçue et étudiée comme un gros mot. Elle pouvait être étroitement associée au juron, si bien que certaines éditions de textes avaient remplacé les gros mots par des pointillés de façon à les passer sous silence ${ }^{5}$. Cet excès de langage était au mieux inclus dans les péchés de la langue et analysé comme l'objet d'un contrôle social confié en priorité à l'Église, avant que Louis IX ne s'en empare quand il édicte son ordonnance sur le blasphème en 1254, ordonnance répétée et amplifiée de nombreuses fois par ses successeurs ${ }^{6}$. La mention d'injures et d'insultes dans les textes était aussi considérée comme une forme de trivialité plutôt réservée au peuple, aux populations d'en bas. La preuve en était dans les chartes urbaines, pour la plupart datées du XII ${ }^{\mathrm{e}}$ siècle, qui mentionnent l'injure comme un élément de trouble de l'ordre public, passible d'une amende, signe par conséquent des débordements d'une société violente comme de l'archaïsme de la justice médiévale qui peinait à la civiliser. Les femmes du peuple n'en étaient pas exemptes, voire même concernées au premier chef à en juger par les sanctions quand elles sont rapportées.

\footnotetext{
${ }^{4}$ Charte communale d'Amiens citée par Jean Boca, La justice criminelle de l'échevinage d'Abbeville au Moyen Âge, 1184-1516, Lille, Émile Raoust, 1930, p. 202 ; Paris, AnF, JJ 155, pièce 132, juillet 1400 .

${ }^{5}$ C'est le cas du Registre criminel du Châtelet de Paris, du 6 septembre 1389 au 18 mars 1392, éd. Henri Duplès-Agier, Paris, Lahure, 1861-1864, 2 vol. Voir par exemple t. 1, p. 337. ${ }^{6}$ Corinne Leveleux-Teixeira, La parole interdite. Le blasphème dans la France médiévale, $X I I I^{e}-X V I^{e}$ siècle : du péché au crime, Paris, De Boccard, coll. «Romanité et modernité du droit », 2001.
} 
À partir des années 1980, l'alliance entre 1'histoire, la littérature et l'anthropologie a peu à peu permis de comprendre que la société médiévale tout entière est une société de l'honneur où la parole et les gestes peuvent rapidement glisser vers le défi et entraîner la vengeance. Le déploiement social de l'injure a aussi interdit de la cantonner au groupe des laboureurs ou des serviteurs, voire des marginaux, et de lui accoler le qualificatif de " grossier », ou alors la société médiévale dans son ensemble est grossière! Comme le montrent les textes évoqués ici, les nobles de la chanson de geste autant que les non-nobles des Marches d'Ancône jouent de l'injure et de l'insulte en reprenant les mêmes mots, les mêmes gestes, les mêmes formes de transgression, en majorité à connotation sexuelle, allant de l'adultère de la «pute reine » à l'homosexualité savamment distillée pour diffamer le héros, mais aussi, ailleurs, du geste du verre froissé qui signe l'incivilité, à la figue blessant le Christ de son outrage. L'injure y apparaît comme un élément des comportements sociaux qu'il convient d'étudier dans son contenu et dans son déroulement pour lui donner un sens.

Restituer au monde d'en bas son honneur, le comparer avec celui de l'aristocratie en concevant l'injure du peuple comme un défi ${ }^{7}$, a été et est encore un long cheminement historiographique, mais il ne saurait suffire. Il importe en effet de comprendre que ce basculement dans la violence du verbe ou du geste, par ailleurs très régulée, s'inscrit dans une stratégie qui met en jeu les intérêts des protagonistes. Autrement dit, il y a un avant de l'injure. Il est difficile à cerner dans les textes judiciaires tels que j'ai pu les étudier, car le récit du crime

\footnotetext{
${ }^{7}$ Je me permets de renvoyer à Claude Gauvard, « Le défi aux derniers siècles du Moyen Âge : une pratique entre guerre et vengeance ", dans Relations, échanges, transferts en Occident au cours des derniers siècles du Moyen Âge. Hommage à Werner Paravicini. Actes du colloque de Paris (4-6 décembre 2008), dir. Bernard Guenée et Jean-Marie Moeglin, Paris, Académie des Inscriptions et Belles Lettres, 2010, p. 383-405, où j'édite cette lettre de rémission destinée à deux frères de Bucy-le-Long, typique d'un honneur blessé par l'injure : ils avaient vengé leur sœur appelée «putain ribaude » par un dénommé Longuepance, car elle aurait été « moult reculee de mariage », Paris, AnF, JJ 120, pièce 33, avril 1381, lettre adressée au bailli de Vermandois.
} 
dans les lettres de rémission a tout intérêt à gommer la préméditation pour faciliter le pardon royal. Néanmoins, le lien est étroit entre la vengeance et le premier antécédent du crime, dans un tiers des cas sous le règne de Charles VI, et dans plus de $80 \%$ des cas pour le crime lui-même ${ }^{8}$. On peut évoquer les haines recuites, mais quels en sont les motifs ? Les biens, les femmes, sans aucun doute. Celui qui est menacé dans ses biens - son honneur matériel annonce alors publiquement à son adversaire « qu'il le courroucerait du corps", ce qui implique injures renouvelées et insultes au sens militaire d'assaut, car la préparation ne fait aucun doute quand on se munit de pourpoints, de haches et de dagues pour attendre son «haineux ». L'injure et l'insulte ne sont alors que l'expression dernière du conflit et elles permettent le basculement dans la violence. Elles sont un défi qui brise le silence d'un contentieux larvé. Par exemple, la lourdeur de dettes anciennes et déshonorantes peut ainsi éclater et se transformer en injures d'ordre sexuel, proférées en public et conduire à la rixe finale ou à un meurtre prémédité ${ }^{9}$. L'honneur a bien été blessé, mais son expression n'a finalement que peu de rapports avec la cause réelle du conflit. Pour se régler, le contentieux, parfois profondément ancré, a besoin de cette expression verbale ou gestuelle qui porte déshonneur à l'adversaire. Dans cette société de l'honneur, l'injure et l'insulte offrent un langage performatif qui permet de résoudre des antagonismes profonds, voire indicibles.

De ce fait, le procédé permet de dominer ou de tenter de dominer l'adversaire en s'insurgeant par la parole. Ce peut être le cas dans le déroulement d'une action violente, insurrectionnelle. En 1410, les moines de l'abbaye de Saint-Denis sont venus faire esclandre dans la cathédrale Notre-Dame de Paris à propos de la calotte crânienne du saint que les chanoines prétendaient posséder comme relique, alors que les moines, en ayant le chef

\footnotetext{
${ }^{8}$ Claude Gauvard, De grace especial. Crime, État et Société en France à la fin du Moyen Âge, Paris, Publications de la Sorbonne, 1991, 2 vol., p. 756, voir aussi chapitres 16 et 17.

${ }^{9}$ Julie Claustre, «La dette, la haine et la force : les débuts de la prison pour dette à la fin du Moyen Âge », Revue Historique, n644, 2007, p. 797-821.
} 
entier du saint décapité, soutenaient posséder le corps dans sa totalité, soit un bien inestimable. Le récit des avocats montre que gestes et paroles se sont multipliés dans la cathédrale jusqu'à en venir aux mains. En retour, l'église de l'abbaye a été placardée d'affiches injurieuses ${ }^{10}$. Les traités savants pour justifier que le corps entier de saint Denis était à l'abbaye et que sa tête avait été décapitée et non décalottée sont venus ensuite, à la demande de la justice. Les clercs - et ici non des moindres - ne sont donc pas les derniers à utiliser ces moyens de persuasion fondés sur la force. Il leur importait de défendre une relique insigne dont la présence, dans leur trésor, était source de rayonnement, mais aussi de revenus substantiels. L'honneur n'est pas que réputation, il se fonde sur les biens et la richesse. L'injure s'inscrit donc dans des compétitions fondées sur des appétits qui opposent les individus, ici deux communautés religieuses, ailleurs deux hommes se disputant des femmes ou des héritages, ou encore une place auprès d'un prince ou d'un seigneur.

$\mathrm{Au}$ Parlement, cette compétition est inhérente à la rhétorique des avocats. La diffamation est constante dans leur bouche. Elle fait partie d'une rhétorique destinée à «tuer » l'adversaire. Reprenons le fameux procès fait à Blanche d'Overbreuc en 1449, coupable d'avoir participé au meurtre de son mari, Guillaume de Flavy $^{11}$. Les avocats des héritiers de Flavy contestent au Parlement la lettre de rémission que Charles VII a accordée à Blanche et ils n'hésitent pas à injurier sa personne en niant ses origines sociales. Vicomtesse d'Acy, héritière du château de Nesles, elle est présentée soit comme la fille de cordonniers ou couturiers, soit d'un charbonnier, ou encore comme bâtarde

\footnotetext{
${ }^{10}$ Sur ce procès qui, malgré les injures et les coups portés, figure dans les registres du Parlement civil, voir Claude Gauvard, «Écrire l'histoire et restaurer l'honneur au Parlement de Paris. À propos de la relique du chef de saint Denis en $1410 »$, dans Rerum gestarum scriptor. Histoire et historiographie au Moyen Âge, Mélanges Michel Sot, dir. Magali Coumert, Marie-Céline Isaïa, Klaus Krönert et Sumi Shimahara, Paris, Presses de l'Université Paris-Sorbonne, 2012, p. 509-519.

${ }^{11}$ Pièces du procès éditées par Pierre Champion, Guillaume de Flavy, capitaine de Compiègne. Contribution à l'histoire de Jeanne d'Arc et à l'étude de la vie militaire et privée au XV siècle, Paris, Honoré Champion, 1906 [réimpr. Genève, 1975].
} 
puisque sa mère se serait accointée d'un prêtre, tout cela dans le plus grand désordre ; quant à son « gouvernement», il aurait laissé à désirer car elle

estoit fort sur sa bouche, mesmement au regard de boire. Et souvent, elle estant a table, quant avoit bien beu, elle retenoit du vin en sa bouche et le gectoit es visaiges de ceulx qui estoient presens : et apres aloit pisser comme ung homme contre ung mur, toute debout, sans aucune vergoigne ${ }^{12}$.

Pourquoi tant de haine? En quoi cette rhétorique est-elle utile, voire nécessaire, au déroulement du conflit? L'injure et la diffamation de l'adversaire ont au tribunal une fonction de catharsis. Elles rendent la haine visible publiquement en lui donnant les mots pour s'exprimer. Autrement dit, elles permettent de commencer à résoudre le conflit et de préparer à un éventuel accord. Nombre de procès au Parlement se terminent par des sentences interlocutoires et les affaires disparaissent parfois de la sphère judiciaire. Archives perdues? C'est possible. Mais les affaires peuvent aussi s'être prolongées par des accords entre parties, tout simplement parce que le face à face verbal a donné lieu à des échanges qui ont déjà vidé une partie de la querelle. Le tribunal est un lieu où l'injure transforme l'adversaire le temps d'une métamorphose suffisante pour satisfaire la partie lésée. C'est dire tout le poids de la parole qui peut, en ces instants, se faire verbe et incarnation du corps, allant, par son contenu, jusqu'à la métamorphose de l'autre. Il devient réellement «fils de putain » et sa mère glisse dans l'infamie de la prostitution, ou bien fils de cordonnier et se rabaisse au rang des « sans nom ${ }^{13}$ ». Le jeu de la domination cher à Bourdieu joue à plein, mais il passe par la transgression des normes susceptible de faire exploser les tabous en même temps que les conventions de langage. Il s'agit de procéder à l'avilissement de l'adversaire

\footnotetext{
${ }^{12}$ Ibid., p. 239-240 et p. 246-247.

${ }^{13}$ Giacomo Todeschini, Au pays des sans-nom. Gens de mauvaise vie, personnes suspectes ou ordinaires du Moyen Âge à l'époque moderne, Lausanne, Verdier, 2015.
} 
avant de demander son exclusion le plus souvent fictive car, comme on le sait, l'affaire se termine au pire par une amende honorable et profitable.

La violence des mots n'est donc pas gratuite et si elle peut être considérée comme symbolique, elle est intentionnelle et efficace. Elle l'est tout autant chez les clercs en mal de réforme politique lorsqu'ils s'adressent aux princes et, de façon générale, chez les écrivains jouant de la dérision pour mieux fustiger les écarts à la norme sociale. Pour les uns, c'est un devoir, pour les autres, c'est un moyen de faire rire, mais le procédé est le même : savoir utiliser à bon escient le «mot qui tue ${ }^{14} »$. Les auteurs de nouvelles jonglent avec la brutalité langagière et l'interprétation de leurs propos doit être soigneusement décodée : il s'agit le plus souvent de dénoncer l'ascension sociale d'un parvenu, la domination de la femme dans le couple, les méfaits de l'adultère... Quant aux conseils donnés aux princes, ils peuvent prendre la forme d'une invective. Mais elle n'a rien à voir avec l'injure au roi qui peut se terminer sous l'épée du bourreau ${ }^{15}$. Elle avance masquée. Pour critiquer les effets d'une grâce royale qu'il juge trop libérale et défendre l'idée que les crimes capitaux, dont l'homicide, sont irrémissibles, Jean Gerson donne la parole au fou du roi. C'est une manière de biaiser pour asséner la vérité : n'est-ce pas le propre du fou d'enrober ses propos les plus subversifs dans la plus grande dérision? Ce qui n'empêche pas le fou de prendre lui-même toutes les précautions pour éviter le courroux royal tant le contenu est grave :

J'ay ouy reciter d'un saige fol qui estoit en la presence du roy quand on luy demandoit grace pour ung homme qui avoit tué ung aultre. Le roy respondoit que par plusieurs fois il avoit fait semblable grace a cest homme et que plus ne la feroit. Lors le fol dist au roy : «Sire, vrayement vous pouves bien faire grace a cest homme, car il n'a pas tué l'autre ». « Pourquoy, dit

\footnotetext{
${ }^{14}$ Le mot qui tue. Une histoire des violences intellectuelles de l'Antiquité à nos jours, dir. Vincent Azoulay et Patrick Boucheron, Paris, Champ Vallon, 2009.

${ }^{15}$ Jacqueline Hoareau-Dodinau, Dieu et le Roi. La répression du blasphème et de l'injure au roi à la fin du Moyen Âge, Limoges, PULIM, 2002.
} 
le roy et qui ha ce fait doncquez?» « Je vous le dirois, respondit le fol, se vous n'en devrez estre courroucié $»$. «Nenny» dit le roy. «Et je vous le dis, Sire, que c'estes vous qui l'avez tué, car se vous eussiez pieça fait justice de cest homme icy, l'autre ne fust point mort ${ }^{16}$.

Ces procédés verbaux employés dans l'espace public ne sont pas le seul mode de résolution des conflits. Il peut être demandé par les autorités judiciaires urbaines ou royales - le Parlement par exemple - de procéder fréquemment à un asseurement selon la coutume du pays. Contrairement à ce qui a été longtemps pensé, cette pratique se prolonge bien au-delà du XIII ${ }^{\mathrm{e}}$ siècle, mais son importance aux derniers siècles du Moyen Âge est encore mal connue. Il s'agit de jurer de ne plus s'agresser verbalement et de ne plus se porter de coups entre individus ennemis, mais aussi entre groupes de parents et d'amis. L'asseurement est un contre-pouvoir apporté à l'injure et à l'insulte. Il repose sur le respect de la parole donnée par serment et il met en jeu les solidarités dans lesquelles l'individu est encore profondément inséré au $\mathrm{XV}^{\mathrm{e}}$ siècle. Malheureusement, on ne le voit surtout se manifester que lorsqu'il a été enfreint, ce qui fausse considérablement l'ampleur de son efficacité.

Toutes les formes d'incitation à la paix entre les parties, parmi lesquelles on peut compter les lettres de rémission car elles soulignent régulièrement l'accord qui a précédé et qui doit succéder à la décision royale, sont un frein à la violence que véhiculent l'injure et l'insulte. Sont-elles suivies d'effet? À quel prix se négocie la «satisfaction faite à partie » ? Une cérémonie rituelle comme l'amende honorable au cours de laquelle le repentant, en tenue de pénitent, crie «merci » et peut dire qu'il a « injurié à tort», suffit-elle à réparer l'honneur blessé ? La présence du public lors de ces cérémonies est-elle un élément suffisant pour restaurer la fama? Et le roi lui-même qui, en héritier des pouvoirs

\footnotetext{
${ }^{16}$ Jean Gerson, «Contre Charles de Savoisy », Euvres complètes, éd. Palémon Glorieux, t. 7, Paris/Tournai/Rome/New York, Desclée et Cie, 1966, p. 338-339. Autre version dans le Vivat Rex, ibid., t. 7, p. 1174.
} 
de l'empereur et du pape, remet d'autorité le suppliant criminel à sa fama en lui accordant sa grâce, apporte-t-il une garantie efficace ?

Ces questions posées n'ont pas toujours de réponses satisfaisantes, mais elles ouvrent sur un foisonnement de solutions possibles pour effacer les effets de l'injure et de l'insulte. Le temps est encore au bricolage parce que l'honneur est une force vive de la société et que la parole qui le véhicule reste un verbe difficile à assourdir, et à plus forte raison à oublier. 
